\title{
Efficient and stable locomotion for impulse-actuated robots using strictly convex foot shapes
}

\author{
Fabio Giardina, and Fumiya Iida, Senior Member, IEEE
}

\begin{abstract}
Impulsive actuation enables robots to perform agile manoeuvres and surpass difficult terrain, yet its capacity to induce continuous and stable locomotion have not been explored. We claim that strictly convex foot shapes can improve impulse effectiveness (impulse used per travelled distance) and locomotion speed by facilitating periodicity and stability. To test this premise, we introduce a theoretical two-dimensional model based on rigidbody mechanics to prove stability. We then implement a more elaborate model in simulation to study transient behaviour and impulse effectiveness. Finally, we test our findings on a robot platform to prove their physical validity. Our results prove, that continuous and stable locomotion can be achieved in the strictly convex case of a disc with off-centred mass. In keeping with our theory, stable limit cycles of the off-centred disc outperform the theoretical performance of a cube in simulation and experiment, using up to 10 times less impulse per distance to travel at the same locomotion speed.
\end{abstract}

Index Terms-Locomotion, Impulsive actuation, Dynamics, Underactuated Robots.

\section{INTRODUCTION}

Actuation in hopping and jumping robots presents one of the major challenges to build efficient and robust hopping machines. Raibert introduced hopping and running robots using hydraulics, compressed air [1], and combustion engines to supply the required forces for high-speed and robust locomotion [2]. These methods, however, require heavy actuators or need to be remotely supplied with energy. Animals appear to avoid this disadvantage by employing spring-like behaviour in muscles and tendons [3], [4], which has inspired engineers to develop smarter actuation mechanisms such as series [5] or parallel elastic actuation [6].

Recently, there has appeared a trend of research that studies hopping and jumping robots with impulsive actuation. Elasticity-stored impulses (ESI) are used in robots like the EPFL jumper [7] where a rapid change from standing to ballistic motion is realised with a 4 bar linkage and a torsion spring. Based on a similar design, the robot Salto [8] is furthermore capable of redirecting its posture during flight phase for a controlled second jump, easily timed due to the impulsive nature of the actuation. These types of jumping robots are considered beneficial for celestial exploration [9].

Based on a different mechanism, momentum-stored impulse (MSI) actuators store impulsive energy in a rotating flywheel. The robot Cubli [10] uses the MSI to lift a cube lying on its face onto one of its edges or vertices, using the flywheel thereafter to balance the cube. Space roboticists have exploited

The authors are with the Engineering Department, University of Cambridge, Cambridge CB21PZ, U.K. (e-mail:ffg20@cam.ac.uk; fumiya.iida@eng.cam.ac.uk). this actuation method for exploratory ventures in unknown environments [11], using impulses for locomotion in lowgravity environments. Similarly, the 3D M-blocks [12] make use of angular impulses to overcome the magnetic potential of their locking mechanism and reconfigure themselves into new structures.

The obvious advantage of impulses is their ability to propel a system over great obstacles as demonstrated for instance by the sand flea robot [13]. Exploiting the accuracy of impulsive actuation furthermore helps to plan a precise landing point. Space robots exploit this property to reach targets in complex and unknown environments by planning a sequence of jumps. A more subtle but no less important advantage of impulsive actuation is its intuitive effect: an impulse applied at a specific point in time changes a system's velocity instantaneously. No integration of the system dynamics needs to be performed to know the outcome of an actuation. This property enables fast trajectory prediction and therefore control decisions to be made, which is shown in the agile motion of Salto [8].

Despite the importance of impulsive actuation, the approach was explored neither for periodic locomotion nor for economical use of impulses in hopping and jumping robots. To maintain or improve locomotion speed given an impulse, it is important to control the stance phase dynamics. The simple and intuitive actuation with impulses should, however, not be tainted with complex sensory feedback. Ringrose [14] argues that a robot with a curved foot can be self-stabilised, meaning that no active feedback control is necessary for stable locomotion. Based on this principle, we have been exploring the influence of strictly convex foot shapes on hopping locomotion in robots like Cargo [15]. We found not only that stable and periodic locomotion can be achieved without any feedback control, but also that we can greatly decrease the energetic cost of locomotion. The intuitive stabilising properties of strictly convex shapes therefore lend themselves as an ideal match for impulsive actuation.

In this paper, we explore the effect of strictly convex foot shapes on the locomotion performance of impulse-actuated systems in terms of speed and economic use of impulses, and compare the results to the theoretical performance of an MSI actuated cube. We first present tools to understand the effect of shape on the dynamics in a theoretical model, and then study the predictions in the case of a disc with an off-centred mass actuated by MSI in simulation. We further present a real world implementation with the robot Robbit to validate our findings.

The remaining paper is structured as follows: Section 2 introduces the modelling approach and the control for loco- 
motion with impulsive actuation and strictly convex shapes. Section 3 presents methods used to find limit cycles, proof of stability, and the results of the system's locomotion performance. Section 4 introduces the robot Robbit, designed using MSI actuation and a strictly convex shape of a disc with an off-centred mass. Experimental results and comparison with the simulation model are presented in section 5. Section 6 discusses the results, and section 7 concludes the paper.

\section{LOCOMOTION MODEL}

In this section, the mathematical methods used to model the hopping locomotion with strictly convex foot shapes is described. After defining shape and the ground contact dynamics in Subsection II-A and II-B, we outline a control and actuation method to induce a flight phase through rotational impulses in II-C. The locomotion model presented here is defined using standard planar rigid body dynamics. That is, the rigid body considered is completely defined by its total mass, moment of inertia with respect to the centre of mass, the centre of mass, and its boundary or strictly convex shape $s(\varphi)$, which defines the set of points of interaction with the environment as a function of the angular parameter $\varphi$ which is the angle relative to the body-fixed frame of reference $S$, as also depicted in Figure 1. Strict convexity, which is a property of the shape $s$ if $\boldsymbol{s}^{T} \frac{d^{2} s}{d \varphi^{2}}<0 \quad \forall \varphi \in[-\pi, \pi]$, is required to distinguish the studied systems from other convex shapes such as polygons and shapes with straight segments, which cannot roll without impacts. We furthermore assume a planar model, as the presented locomotion mode does not depend on the lateral dimension.

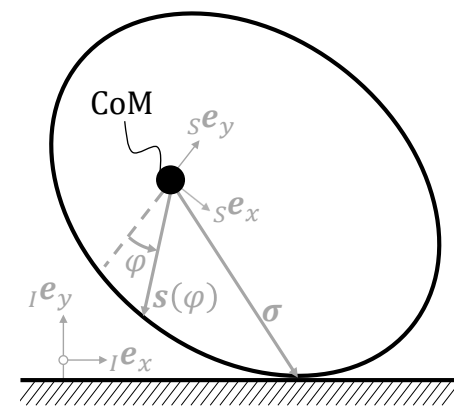

Fig. 1: Definition of shape $s$, which is a function of the parameter $\varphi$ with respect to the body-fixed frame of reference $S$ located at the centre of mass (CoM) of the body, and the contact function $\sigma$.

\section{A. Constrained equations of motion}

We would like to point out that we assume a no-slip condition at this point to simplify analysis. As explained in more detail later, the results presented in Section III-C and $\mathrm{V}$ will consider friction of the Coulomb type for the prediction of robot performance. We implement interactions by specifying kinematic constraints a priori, and solve the resulting equations to find the enabling forces. We will denote with $\boldsymbol{q}=(x, y, \phi)^{T}$ the generalised coordinates with $x$ and $y$ the coordinates of the centre of mass with respect to the inertial frame of reference $I$ located at origin $O$ and $\phi$ the angular rotation of the body fixed frame of reference $S$ with respect to $I$. We enforce the rolling constraint by requiring that the relative velocity between the ground contact point on the robot and the ground vanishes, i.e.

$$
\mathbf{J}_{C} \dot{\boldsymbol{q}}=\left(\begin{array}{c}
\dot{x}-\sigma_{y}(\phi) \dot{\phi} \\
\dot{y}+\sigma_{x}(\phi) \dot{\phi}
\end{array}\right)=\mathbf{0},
$$

with $\mathbf{J}_{C}$ the Jacobian of the ground contact and $\dot{\boldsymbol{q}}$ the generalised velocity. We will refer to the vector function $\boldsymbol{\sigma}=\left(\sigma_{x}, \sigma_{y}\right)^{T}$ as contact function, as it represents the vector pointing from the centre of mass to the current contact point. After defining the kinematic constraints on acceleration level, we can use Gauss's principle to enforce the constraints via Lagrangian multipliers in the equations of motion. The equations of motion of the constrained system with possible external force $\boldsymbol{F}$, that includes also the gravitational force, read

$$
\mathbf{M} \dot{\boldsymbol{u}}=\boldsymbol{F}+\mathbf{J}_{C}^{T} \boldsymbol{\lambda}
$$

where $\mathbf{M}$ is the generalised mass matrix, and $\boldsymbol{u}=\dot{\boldsymbol{q}}$ the generalised velocity. The Lagrange multipliers are found by combining (2) and the derivative of (1)

$$
\boldsymbol{\lambda}=-\left(\mathbf{J}_{C} \mathbf{M}^{-1} \mathbf{J}_{C}^{T}\right)^{-1}\left(\mathbf{J}_{C} \mathbf{M}^{-1} \boldsymbol{F}+\boldsymbol{\xi}\right),
$$

with $\xi$ the gyroscopic terms which arise due to the kinematic constraints.

\section{B. Impulsive transitions}

We employ a Newtonian kinematic impact law in normal direction with coefficient of restitution $\varepsilon=0$ to model inelastic impacts. We model tangential impacts also with a coefficient of restitution $\varepsilon=0$ and assume a no-slip condition. This avoids the effects of friction and tangential compliance and simplifies the governing dynamics of the locomotion mode presented in the next subsection. More specifically, we require during this impulsive event

$$
\dot{\boldsymbol{g}}^{+}+\mathbf{E} \dot{\boldsymbol{g}}^{-}=\mathbf{0},
$$

where $\mathbf{E}=\mathbf{0}$ is the diagonal matrix of coefficients of restitution, $\dot{\boldsymbol{g}}=\mathbf{J}_{C} \dot{\boldsymbol{q}}$ is the contact point velocity, and we use the superscripts + and - to indicate post-contact and pre-contact conditions, respectively. The requirement (4) then leads to a post-contact velocity

$$
\boldsymbol{u}^{+}=\left(\mathbf{I}-\mathbf{M}^{-1} \mathbf{J}_{C}^{T}\left(\mathbf{J}_{C} \mathbf{M}^{-1} \mathbf{J}_{C}^{T}\right)^{-1} \mathbf{J}_{C}\right) \boldsymbol{u}^{-},
$$

which defines the transition behaviour from unconstrained to constrained equations of motion in terms of velocity. Note that the transition of generalised coordinates $\boldsymbol{q}$ is smooth and does not change over the impulsive event.

Impact modelling has a long history and many models come with inconsistencies in certain situations often due to the rigid body hypothesis. For a coefficient of restitution $\varepsilon=0$, which is the case in this work and was tested in our previous investigations [6] and [15], the Newtonian kinematic impact law provides equivalent results to alternatives like Poisson's 


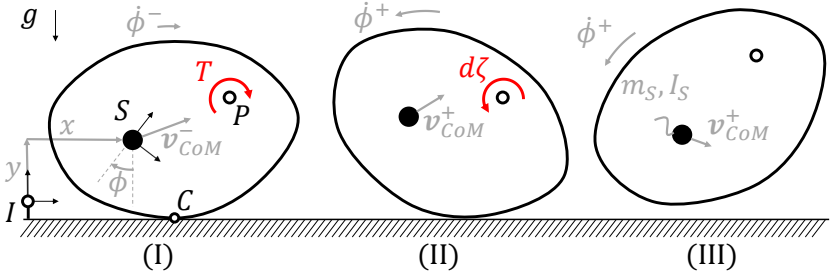

Fig. 2: Locomotion of arbitrary strictly convex shape through momentum-stored impulse. (I) Rigid body with centre of mass $S$ and generalised coordinates $\boldsymbol{q}=(x, y, \phi)^{T}$ is actuated with a constant torque $T$ around arbitrary point $P$. The current angular velocity $\dot{\phi}^{-}$is such that the system rolls instantaneously around contact point $C$, causing a centre of mass velocity $\boldsymbol{v}_{C o M}^{-}$. (II) An angular impulse $d \zeta=T \times t_{S}$, with $t_{S}$ the stance phase time, instantaneously changes the angular velocity to $\dot{\phi}^{+}$and induces a flight phase. Note that the centre of mass translational velocity is not affected directly by the angular impulse. (III) During flight phase, no torque is applied. The system with mass $m_{S}$ and moment of inertia around the centre of mass $I_{S}$ is only influenced by gravitational acceleration $g$.

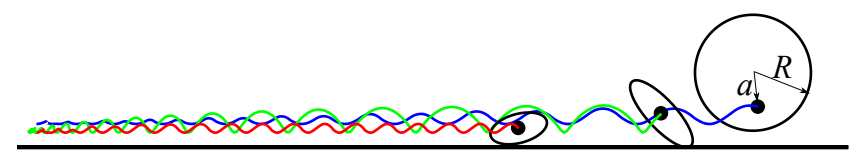

Fig. 3: Diversity of hopping behaviour for different shape functions in simulation. The blue trajectory corresponds to the off-centred disc with radius $R=0.2 \mathrm{~m}$ and eccentricity $a=R / 2$, the green trajectory to an ellipse with semi-major axis $0.75 R$ and semi-minor axis $0.25 R$, and the red one for an ellipse with semi-major axis $0.5 R$ and semi-minor axis $0.25 R$. Actuation for all three cases is $T=0.38 \mathrm{Nm}$ and impulse limit $d \zeta_{\text {lim }}=0.052 \mathrm{Nms}$.

or Stronge's energetically consistent impact law even for noncollinear collisions with friction [16]. This will be especially important in the extension of this model with friction as will be discussed in Section III-C.

To transition from constrained to unconstrained equations of motion we do not require any kinematic conditions, except that the impulsive force applied to the system is causing the ground contact point to have a velocity component which is pointing away from the ground. In other words, if we apply an impulse $d \boldsymbol{\zeta}$, we only need to satisfy the condition

$$
{ }_{i} \boldsymbol{e}_{y}^{T} \mathbf{J}_{C} \boldsymbol{u}^{+}={ }_{I} \boldsymbol{e}_{y}^{T} \mathbf{J}_{C} \mathbf{M}^{-1} d \boldsymbol{\zeta}+{ }_{I} \boldsymbol{e}_{y}^{T} \mathbf{J}_{C} \boldsymbol{u}^{-}>0,
$$

where ${ }_{I} \boldsymbol{e}_{y}$ is the unit vector in vertical direction of the inertial frame of reference $I$, as indicated by the left subscript.

\section{Locomotion with strictly convex shapes}

We now describe the method for locomotion using strictly convex shapes. Inertial properties of the system are mass $m_{S}$ and moment of inertia $I_{S}$ with respect to the centre of mass. The position of the centre of mass is indicated by the generalised coordinates $x$ and $y$, in horizontal and vertical direction, respectively. We apply a torque $T$ in an arbitrary point $P$, as indicated in Figure 2 (I), causing an accelerated motion in form of rolling around contact point $C$. The centre of mass trajectory of the system is constrained by the shape and is always perpendicular to the contact function $\sigma$ during stance phase. Note that due to the strict convexity of the shape, the body rolls over the ground without any collisions.

To induce flight phase, we need to apply an impulsive force as defined by (6). Here we come to the central property of this mode of locomotion. The torque $T$ which has accelerated the system must have generated the angular momentum $\Delta L=$ $T t_{S}$ as a result of the reaction torque in point $P, t_{s}$ being the time for which the constant torque $T$ was applied to the body. We exploit this angular momentum and assume that we can transfer it in an inelastic collision to the main structure. A possible outcome of such an event is indicated in Figure 2 (II). The impulse causes an angular velocity which is opposite in direction to the stance phase, hence causing a backward rotation which leads to a flight phase (III).

We assume the reaction torque has acted on a flywheel located in point $P$ with moment of inertia $I_{R}$ with respect to $P$. The angular velocity of the flywheel after time $t_{S}$ under constant torque $T$ is then

$$
\dot{\phi}_{R}^{-}=\dot{\phi}_{R, 0}+\frac{T t_{S}}{I_{R}},
$$

With $\dot{\phi}_{R, 0}$ its initial angular speed. For the transfer of impulse, we exploit conservation of angular momentum which results in an expression for the post-impact angular velocity of the main body

$$
\dot{\phi}^{+}=\frac{\dot{\phi}^{-} I_{S}+\dot{\phi}_{R}^{-} I_{R}}{I_{S}+I_{R}} .
$$

The transferred impulse is then simply derived by

$$
d \zeta=I_{S}\left(\dot{\phi}^{+}-\dot{\phi}^{-}\right) .
$$

We can now define a control method to induce hopping in strictly convex shapes with momentum-stored impulse actuation. We define the control parameters constant torque $T$ and impulse limit $d \zeta_{\text {lim }}$, and start in stance phase, i.e. with the constrained equations of motion (2) and a system state $\boldsymbol{q}_{0}$ which comply with the ground contact conditions. First, the constant torque is applied onto the system, accelerating both the main body and the flywheel, until the impulse (9) reaches the impulse limit $d \zeta_{\mathrm{lim}}$. Subsequently, momentum between the flywheel and the main body is exchanged, causing a backward rotation and inducing flight phase. No torque is applied during flight phase. Once any shape point coincides with the ground, an impact forces the transition from flight to stance, initiating a new cycle by applying the constant torque $T$.

We have tested this control method for various shape function in simulation as shown in Figure 3, highlighting how this simple control law generates stable locomotion trajectories for randomly chosen strictly convex shapes. Note that the three illustrated cases are controlled with exactly the same control law and inertial parameter values. The maximal speed which can be obtained with the suggested control strategy is arbitrary, 
TABLE I: Parameter ranges for simulation and robot.

\begin{tabular}{|c|c|c|c|}
\hline & Parameter & Symbol & Value \\
\hline \hline \multirow{3}{*}{ Generalized } & Horiz. pos. & $x$ & {$[-\infty, \infty] \mathrm{m}$} \\
coord. & Vert. pos. & $y$ & {$[0, \infty] \mathrm{m}$} \\
& Angle main body & $\phi_{S}$ & {$[-\pi, \pi] \mathrm{rad}$} \\
& Angle flywheel & $\phi_{R}$ & {$[-\pi, \pi] \mathrm{rad}$} \\
\hline \hline & Foot radius & $R$ & $0.2 \mathrm{~m}$ \\
& Eccentricity & $a$ & $0.1 \mathrm{~m}$ \\
& Mass main body & $m_{S}$ & $0.974 \mathrm{~kg}$ \\
Exp. \& simu. & Mass flywheel & $m_{R}$ & $0.431 \mathrm{~kg}$ \\
fixed param. & MoI main body & $I_{S}$ & $3 \cdot 10^{-3} \mathrm{~kg} \mathrm{~m}^{2}$ \\
& MoI flywheel & $I_{R}$ & $1.5 \cdot 10^{-3} \mathrm{~kg} \mathrm{~m}^{2}$ \\
& Gravity & $g$ & $9.81 \frac{\mathrm{m}}{\mathrm{s}^{2}}$ \\
& Coeff. of rest. & $\varepsilon$ & $0[-]$ \\
\hline \hline Exp. control & Torque & $T$ & {$[0.1,0.3] \mathrm{N} \mathrm{m}$} \\
$\beta=\left\{T, d \zeta_{\text {lim }}\right\}$ & Impulse limit & $d \zeta_{\text {lim }}$ & {$[0.04,0.07] \mathrm{Nms}$} \\
\hline
\end{tabular}

as long as there is no torque limit or restrictions due to friction. However, this does not guarantee that we can find a control that leads to a limit cycle at arbitrarily high speeds.

\section{StABILITY OF LOCOMOTION}

The subsequent analysis is applied to a shape defined by a disc with an off-centred mass, where $R$ denotes the radius of the disc, and $a$ stands for the eccentricity of the centre of mass, measured from the centre of the disc. The eccentricity thereby influences the stance phase dynamics and energy loss during collision. See also Figure 3 for an indication of these two parameters. This description simplifies the contact function to

$$
\boldsymbol{\sigma}(\boldsymbol{q})=\left(\begin{array}{c}
-a \sin (\phi) \\
a \cos (\phi)-R
\end{array}\right) .
$$

The numerical parameters of the system are given in Table I. The applied control sequence is as described in the previous section. Note that even though we restrict the shape to a disc with an off-centred mass, the presented procedure is valid for any strictly convex shape function $\sigma$.

In the next step, we investigate if this control strategy, together with the defined contact function (10), can lead to periodic solutions corresponding to continuous locomotion.

\section{A. Finding periodic solutions}

We claim that periodic and continuous motion, i.e. a motion which maps the take-off state back to itself after one cycle of stance and flight phase, improves the performance of impulseactuated systems. If we look at the Poincare section at the pre-take-off position, the system (without the flywheel) can be completely defined by its angular velocity $\dot{\phi}$ and its posture $\phi$ due to the fact that the centre of mass follows a trajectory predefined by the contact function. Given angle and angular velocity at take-off, the trajectory until the next take-off point is completely defined by the control $\beta=\left\{T, d \zeta_{\text {lim }}\right\}$, which describes the set torque and impulse limit. The impulse limit is defined as $d \zeta_{\text {lim }}=\left[0,0, d \zeta_{\text {lim }}\right]^{T}$ pointing in the direction of unit vector $\boldsymbol{e}_{\phi}$. We ask the question whether there is a control configuration $\beta^{*}=\left\{T^{*}, d \boldsymbol{\zeta}_{\text {lim }}^{*}\right\}$, i.e. a torque-impulse limit combination, which maps the system state just before take-off $\boldsymbol{z}_{T O}^{-*}=\left(\phi_{T O}^{-*}, \dot{\phi}_{T O}^{-*}\right)^{T}$ to itself. The subscripts $T O$ and $T D$ indicate take-off and touchdown states, respectively. The state of the flywheel has no influence on the dynamics except at the impulsive event, but we need to make sure that the angular velocity of the flywheel $\dot{\phi}_{R, T O}^{-}$is being mapped back to itself to truly guarantee periodic behaviour.

We describe next the forward dynamics of one cycle to search for fixed-points. Every new iteration starts with the computation of the post-take-off state

$$
\boldsymbol{u}_{T O}^{+}=\mathbf{M}^{-1} d \boldsymbol{\zeta}_{\text {lim }}+\boldsymbol{u}_{T O}^{-},
$$

followed by a mapping of the post-take-off state to the pretouchdown state through the body's ballistic motion given by the implicit equation for the flight time $t_{F}$

$$
y+\dot{y} t_{F}-\frac{1}{2} g t_{F}^{2}+a \cos \left(\phi_{T O}+\dot{\phi}_{T O} t_{F}\right)-R=0 .
$$

The pre-touchdown velocities can then be calculated with

$$
\boldsymbol{u}_{T D}^{-}=\boldsymbol{u}_{T O}^{+}-g t_{F I} \boldsymbol{e}_{y},
$$

which can be mapped to the post-touchdown velocities using (5). The touchdown position in generalized coordinates is then given by the ballistic equations

$$
\boldsymbol{q}_{T D}=\boldsymbol{q}_{T O}^{+}+\boldsymbol{u}_{T O}^{+} t_{F}-1 / 2 g t_{F I}^{2} \boldsymbol{e}_{y}
$$

As pointed out in section II-A, the stance phase is modelled through the kinematic constraints defined by the ground. The case of the off-centred disc is defined by the contact function (10), which renders the constrained equations solely dependent on the quantities $\phi$ and $\dot{\phi}$, i.e. the angular position and its temporal derivative. The constrained equations of motion arising from (2), can be written in a concise ODE in the case of the off-centred disc by replacing $z_{1}=\phi$ and $z_{2}=\dot{\phi}$ to yield

$$
\left[\begin{array}{l}
\dot{z}_{1} \\
\dot{z}_{2}
\end{array}\right]=\left[\begin{array}{c}
z_{2} \\
-\frac{T+m g a \sin z_{1}+m a R z_{2}^{2} \sin z_{1}}{m R^{2}+m a^{2}+I_{S}-2 m R a \cos z_{1}}
\end{array}\right] .
$$

To search for solutions, we use a single-objective constrained nonlinear program implemented in Matlab ${ }^{\circledR}$ using fmincon. We provide initial values of the actuation for the nonlinear solver by assuming the flight phase is symmetric, i.e. $\phi_{S}^{T O}=-\phi_{S}^{T D}$, where the superscripts $T O$ and $T D$ indicate take-off and touchdown conditions, respectively.

As a constraint to the nonlinear solver, we force the objective function to return its value once the initial condition $\phi_{T O}$ is reached. The objective function then solely depends on the other two recurring quantities, and we define the cost as

$$
C=\sqrt{\left(\frac{\dot{\phi}_{0}-\dot{\phi}_{1}}{\dot{\phi}_{0}}\right)^{2}+\left(\frac{d \zeta_{l i m}-d \zeta_{1}}{d \zeta_{l i m}}\right)^{2}},
$$

where the subscripts 0 and 1 indicate the initial state and the state after going through the forward dynamics (11) to (15), respectively. The first squared term in Equation (16) ensures that the angular velocity error is minimised, and the second squared term reduces the flywheel angular velocity error, i.e. the transferred impulse, in the nonlinear optimisation.

The fixed-point actuations found in the nonlinear optimization are shown in Figure 4. The colour indicates the value of 


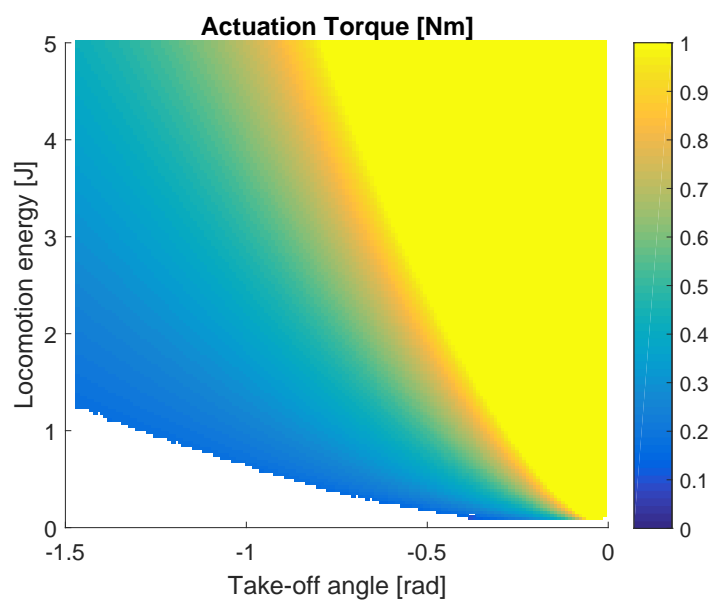

(a)

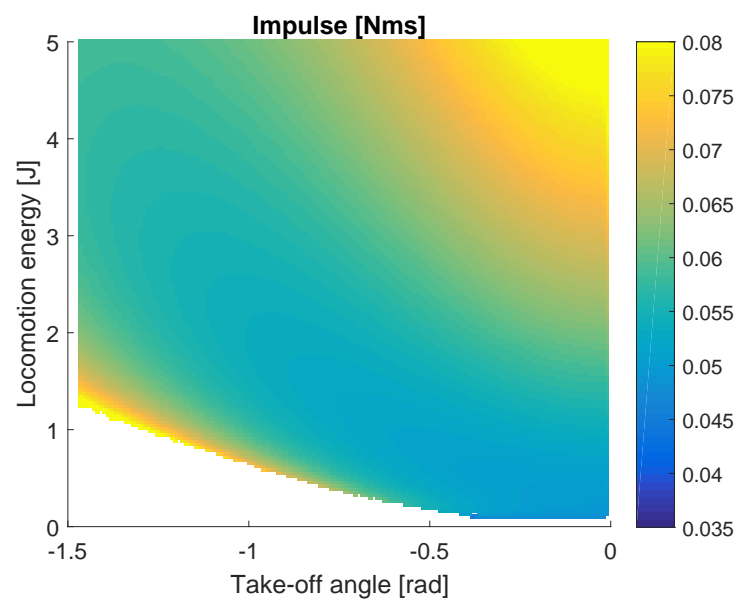

(b)

Fig. 4: Fixed point actuation $\beta^{*}$ found in nonlinear optimization as a function of take-off angle and total energy before take-off. The control is defined by torque (a) $T[\mathrm{Nm}]$ and angular impulse limit (b) $d \zeta_{\text {lim }}[\mathrm{Nms}]$. Note that the results are computed for a 150x150 grid on the shown angle-energy space.

obtained torque in Figure 4a, and impulse limit in Figure 4b, as a function of the fixed-point's take-off angle and locomotion energy. We defined the locomotion energy as the system energy just before take-off, which is proportional to the state $\dot{\phi}_{0}$. White areas indicate that no fixed-point was found. This arose mainly due to the fact that the take-off energy was lower than the potential energy required to reach posture $\phi_{0}$. Figure 4 conveys an important message, which is that fixedpoints exist for the described control method. It means that there exists periodic locomotion for which the torque not only accelerates the flywheel back to its initial speed at the take-off angle, but also makes up for the energy lost during collision, by re-accelerating the main body during stance phase as governed by the ODE (15). In other words, for any point in the state space above the white area there exists a fixedpoint control configuration $\beta^{*}$. The fixed-point torques and impulses transition smoothly in the state space, suggesting that neighbouring fixed-points have a similar behaviour. As we will see in the next section, stability is not guaranteed by this smooth transition. An interpretation of the locomotion behaviour is also possible by looking at the fixed-point actuations: a high torque in combination with a low impulse, as in the bottom right region of the state space, corresponds to a short stance phase. This is because the impulse limit is reached rapidly during stance phase, leading to a high hopping frequency and short stance phase. A high impulse with a low torque, as in the bottom left region, means that stance phase is long because the time to charge the flywheel impulse is long leading to a prolonged stance phase. The proposed control strategy therefore leads to a variety of periodic motions in our proposed locomotion method.

\section{B. Stability of fixed-points}

We have shown that fixed-points exist for the disc with offcentred mass, but the periodic trajectory of the robot is only maintained if it is also stable in the face of disturbances. To study the stability of the found fixed-points, we can look at the linearisation of the concatenation of (11) to (15)

$$
\boldsymbol{z}_{1}=\boldsymbol{\Gamma}\left(\boldsymbol{z}_{0}, \theta\right),
$$

where $\theta$ represents all fixed parameters such as mass and actuation torque.

The linearised map is

$$
\mathbf{A}=\left.\frac{\partial \boldsymbol{\Gamma}}{\partial \boldsymbol{z}}\right|_{\boldsymbol{z}^{*}, \theta^{*}}=\left.\left[\begin{array}{cc}
\frac{\partial \Gamma_{1}}{\partial \phi} & \frac{\partial \Gamma_{1}}{\partial \dot{\phi}} \\
\frac{\partial \Gamma_{2}}{\partial \phi} & \frac{\partial \Gamma_{2}}{\partial \dot{\phi}}
\end{array}\right]\right|_{\phi_{0}^{*}, \dot{\phi}_{0}^{*}, \theta^{*}},
$$

and we make use of the fact that this term dominates the dynamics in a Taylor series expansion for infinitesimally small disturbances $\delta \boldsymbol{z}$. If therefore the magnitude of disturbance decreases for all possible directions over one stance iteration, the nonlinear fixed-point is locally asymptotically stable. This is the same as saying that the absolute value of the eigenvalues of $\mathbf{A}$ has to be smaller than 1, i.e. $\left|\lambda_{i}\right|<1 \forall i \in\{1,2\}$. It is obvious that (17) cannot be represented in a closed expression as it contains differential and implicit equations. We show in the appendix how to overcome these impediments and still be able to obtain an accurate linearisation of (17) around a fixed-point.

We computed the stability for all the found fixed-point actuations (see Figure 4). The coloured points in Figure 5a refer to the absolute value of the maximal eigenvalue of the linearised map in Equation (18), as a function of take-off angle and locomotion energy. We highlight unstable regions, i.e. regions for which $|\lambda|_{\max }>1$, by small black dots. We observe that a large area of the state space is stable, meaning that the fixed-point control method in these points is rejecting small state disturbances. As we will see in section III-B, the system controlled by a stable fixed-point control $\beta^{*}$ not only rejects small disturbances, but converges to the fixed-point even from resting position. We further note that even though the fixed-point control varies smoothly within the state space as seen in Figure 4, there are abrupt stability changes in Figure 


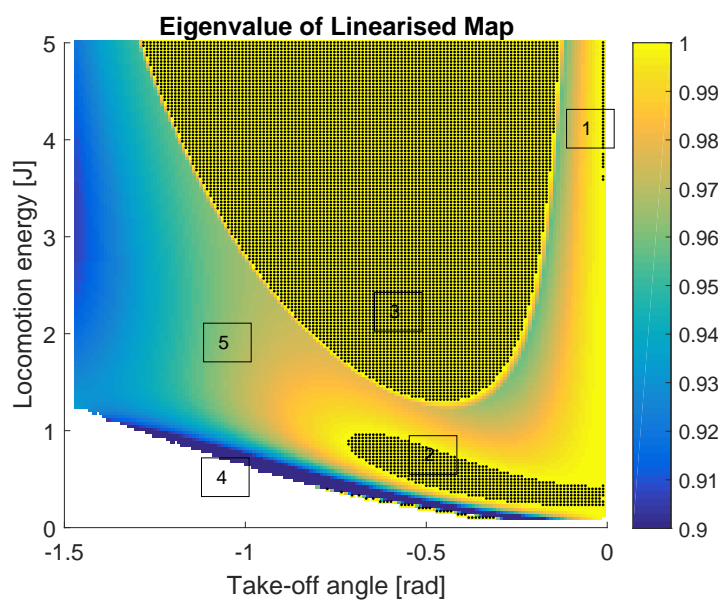

(a)

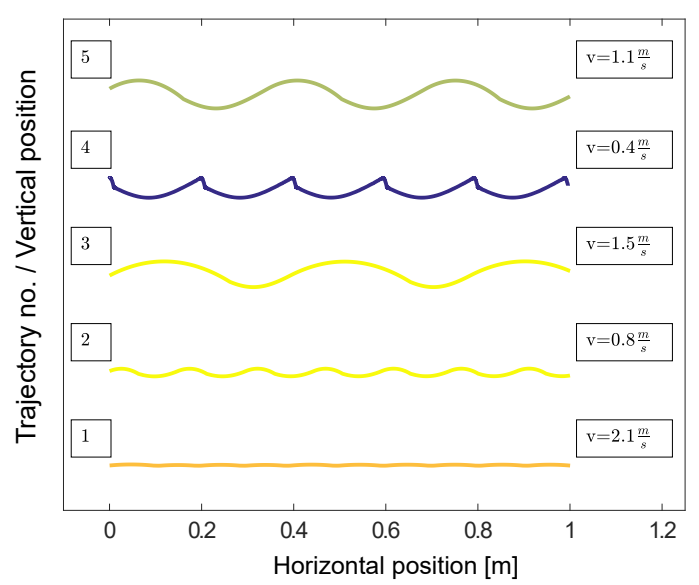

(b)

Fig. 5: Stability of the off-centred wheel model as a function of take-off postures and total energy before take-off. Control parameters for the corresponding fixed-points are given in Figure 4. (a) Maximum absolute value of the eigenvalues of the linearized return map of (17). Unstable fixed-points for which $|\lambda|_{\max }>1$ are marked with a black dot. (b) Centre of mass trajectories for various stable and unstable fixed-points. Velocity on the right hand side is derived by the time taken to reach the $1 \mathrm{~m}$ mark.

$5 \mathrm{a}$, as seen in the transition from the coloured stable to the black dotted unstable area. We analysed the instability and found that the unstable fixed-point actuations converge to a period- 2 cycle when perturbed. This means that even if a fixedpoint is deemed to be unstable, the fixed-point control $\beta^{*}$ still converges to periodic hopping locomotion. The performance of these points has to be questioned, however, as we find that every second impulse reduces rather than increases locomotion speed.

The locomotion trajectories of centre of mass of the disc for some regions are illustrated in Figure $5 b$ together with their locomotion velocity. The motion of trajectory type 1 is operating at high frequency and disappearing hopping height, looking as if the system was sliding on the ground. Bearing in mind that it has set out with the highest locomotion energy, it is also the fastest gait. Motion of type 2 and type 3 are both unstable, yet different in behaviour. While type 2 looks like a sinusoidal trajectory with symmetric flight and stance phase behaviour, type 3 shows more impulsive stance phase properties. The latter particularly looks like running and hopping trajectories of animals at higher speeds. Motion type 4 is the most stable trajectory and shows the least smooth behaviour. It is characterised by a slow final phase during stance, almost halting at the take-off position, followed by a flight phase with vanishing take-off speed in translation, but quick retraction of the shape structure until it hits the ground. The large region of stability characterised by motion type 5 shares smooth and symmetric locomotion trajectories, similar to type 2 .

\section{Performance of fixed-points}

To study the performance of the fixed-point actuation as per Figure 4, we initialised the off-centred disc from resting position. We employ a numerical multibody-dynamics simulation

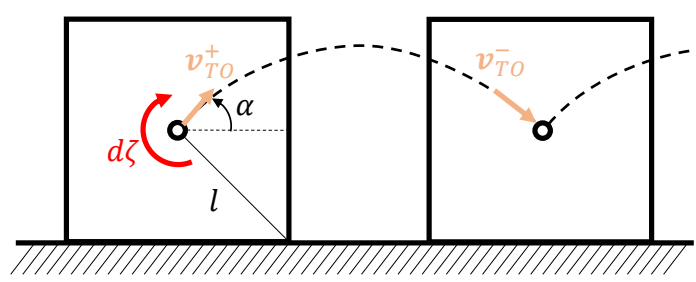

Fig. 6: Simplified hopping model of non-strictly convex shapes. The impulse $d \zeta$ induces the take-off velocity $\boldsymbol{v}_{T O}^{+}$, perpendicular to the diagonal $l$. The shape of the body is only considered at take-off and touchdown, and flight phase is modelled via a ballistic motion of a point mass.

specialised for ground contact modelling with unilateral constraints. We cannot use the analytical approach as we used for the stability analysis because we assumed bilateral constraints of the forward-dynamics, which is only admissible while the constraint forces in normal direction to the ground are larger than zero, i.e. $\lambda_{N}>0$. This condition is violated, when the centrifugal acceleration is larger than the gravitational one, which leads in the bilateral constraint case to the ground pulling the system down, while in a unilateral constraint case, a flight phase is induced. There is a second advantage of using the detailed model, which is that we can simulate a more realistic scenario with friction. Friction is modelled with a Coulomb friction law

$$
\lambda_{T}=\mu \lambda_{N} \operatorname{Sgn}\left(\gamma_{T}\right),
$$

where $\lambda_{T}$ is the tangential friction force at the contact point, $\mu$ is the friction coefficient, $\lambda_{N}$ is the normal force at the ground contact point, $\mathrm{Sgn}$ is the filled-in relay function which 


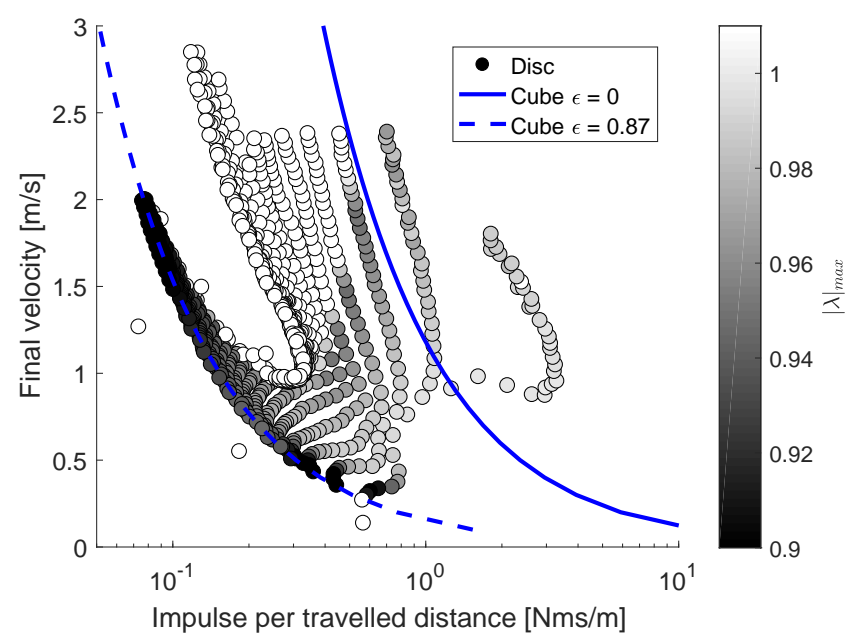

Fig. 7: Locomotion speed as a function of impulse per travelled distance after 20 seconds of simulation starting from resting position of the off-centred disc model. The system is controlled with the found fixed-point actuations in a uniform $30 \times 30$ grid based in the same state space as shown in Figure 4. Brightness indicates the absolute maximal eigenvalue of the fixed-point return map. The solid line indicates the theoretical impulse per travelled distance of a cube shape with inelastic collisions $\epsilon=0$, and the dashed line for a cube with coefficient of restitution $\epsilon=0.87$ as per Equation (21).

is set-valued at $x=0$ and is defined as $\operatorname{Sgn}(-|x|)=$ $-1, \operatorname{Sgn}(|x|)=1$, and $\operatorname{Sgn}(0)=[-1,1], x \in \mathbb{R}$, and $\gamma_{T}$ is the tangential velocity of the ground contact point. The same model has been used and is described in more detail in [6] for another hopping robot. It is largely based on the linear complementarity problem (LCP) formulation of dynamics with unilateral constraints presented in [17] and has been proven to be an effective way to handle contact problems in locomotion systems modelled as rigid bodies with friction and impact, such as shown in the examples paper [18]. We compared the no-slip model as described in Section II-B with the frictional model and found a close correspondence of terminal locomotion speeds for all fixed-point actuations. However, we will continue with the more detailed frictional model from this point onward to ensure that our results are as close to the real system as possible and to monitor the constraint forces. As the LCP can be prone to ill-conditioned events, we also monitored it during simulation and found that no such events occurred at any point.

The system is modelled with two rigid bodies, namely the flywheel and the main body, with properties as described in Table I. The equations of motion of the multibody system are derived using the projected-Newton-Euler approach, and we assumed gravity was the sole constant external force. We assume no damping at this stage in the internal angular degree of freedom and choose a friction coefficient of $\mu=1$. As implied, we start the simulation from a resting position and apply the torque and impulse limit as found by the fixed-point search (see Figure 4) for 30 different fixed-point positions $\phi$ and energies $E_{T O}$. The torque is only being applied during stance phase and only until the flywheel is ready to transfer the impulse $d \zeta_{\text {lim }}$. The simulation runs for 20 seconds with a time step of $d t=2 \times 10^{-4} \mathrm{~s}$ using an Euler integration method.

We compare our results to the theoretical case of a cube actuated with a flywheel as has been studied in [19]. Similar cube-like systems have also been developed and studied in [10] and [12]. We set the inertial parameters of the cube in accordance with the off-centred disc parameters given in Table I. In terms of size, we set the diagonal of the face of the cube to be $l=2(R-a)$, as shown in Figure 6 . The cube lies on one of its faces and we apply an impulse and write the resulting velocity after impulse

$$
\boldsymbol{u}_{T O}^{+}=\boldsymbol{u}_{T O}^{-}+\frac{d \zeta}{m l^{2}+I_{S}}\left(\begin{array}{c}
l \cos \alpha \\
l \sin \alpha \\
-1
\end{array}\right)
$$

with $\alpha$ the take-off angle with respect to the horizontal axis. We justify the assumption that the cube lies face-down at every hop with the long charging time that is required to generate a sufficient impulse which allows the cube to roll around its edge: using the numerical values given in Table I we obtain for the minimal required take-off energy for the cube $E_{T O, \text { cube }} \approx 0.8 \mathrm{~J}$, which is merely the potential energy required to lift the cube on its edge. This energy must be provided with a single impulse, and we find that $d \zeta_{\text {cube }} \approx 0.3 \mathrm{Nms}$, which is 4-8 times larger than the impulses required for the feasible fixed-point controls with the strictly convex shape. Using the maximal feasible torque of $0.3 \mathrm{Nm}$ according to Table I, the cube robot would have to be charged for at least $1 s$ before the required impulse is ready. By the time the impulse is ready, the motion of the cube will have come to rest. This reasoning is also confirmed by the charging time and cube motion of the similar sized cubli robot [10]. We assume a ballistic flight phase, according to the translational initial velocity $\boldsymbol{v}_{T O}^{+}$, during which we ignore the shape of the cube and its rotation. Next, we find the impulse required per travelled distance using (20) together with the restitution law for translational velocities $\boldsymbol{v}_{T O}^{-}=\epsilon \boldsymbol{v}_{T O}^{+}$

$$
\frac{d \zeta}{x}=\frac{g\left(m l^{2}+I_{S}\right)(1-\epsilon)}{2 l u_{x}^{+} \sin \alpha},
$$

with $\epsilon$ the coefficient of restitution, and $u_{x}^{+}$the locomotion speed. This equation corresponds to the additive impulse required to travel a distance $x$ with a sequence of hops with coefficient of restitution $\varepsilon$ for the given cube. We will refer to the quantity $d \zeta / x$ as impulse effectiveness and will call systems that travel with a low $d \zeta / x$ impulse effective. It thus defines a cost of travel in terms of required impulse over a certain distance as a function of locomotion speed. If energy is restored during the stance phase, the coefficient of restitution becomes greater than zero, hence reducing the cost. For the case of an inelastic cube, we set $\epsilon=0$, and $\alpha$ to $\pi / 4$. Note that this simple model is not confined to a cube shape. Any polygonal shape can be studied by adapting the centre of mass to contact point distance $l$, and its corresponding angle $\alpha$. For a linear impulse acting at angle $\alpha$ on a point mass, 
we would obtain $\frac{d \zeta}{x}=\frac{g(1-\epsilon)}{2 u_{x}^{+} \sin \alpha}$, which also emphasises the improvement of impulse effectiveness with locomotion speed.

Figure 7 shows the performance of 900 fixed-point actuations sampled from a uniform 30x30 grid in Figure 4. The simulation ran for a total of 20 seconds. The vertical axis corresponds to the average velocity over the last 10 seconds, and the horizontal axis is the sum of the transferred impulses over the last 10 seconds divided by the distance travelled during the last 10 seconds. The off-centred disc was initialised from resting position. The brightness of each dot indicates its maximum absolute eigenvalue, taken from the results displayed in Figure 5. The results show that the least demanding locomotion actuation in terms of impulse per travelled distance correlates with stability of the theoretical return map. This is understood for the case of white dots, as the instability forces the system to use two impulses per stance for locomotion, as discussed in section III-B. For stable fixed-points, the performance tends to improve the smaller the maximal eigenvalue of the linearised fixed-point. This behaviour is desirable, as it shows that the most efficient modes of locomotion are also the most stable ones. We find that stability increases with the take-off angle, meaning that a long stance phase improves stability. Indeed, we find that the most stable trajectories are of the type 4 and 5 as in Figure $5 b$. Trajectories with short stance phases as compared to their flight phase as in type 3, tend to be unstable. Furthermore, the figure opposes the theoretical findings of the inelastic cube performance to the off-centred disc. We observe that the strictly convex shape of the disc for the most stable actuation can lead to a requirement of impulse per travelled distance ten times lower than for the cube. This dominance of the disc can be explained by its dynamic motion: while the strictly convex shape can build up its kinetic energy over several impulses, the cube as studied here lacks this feature due to the high potential energy required to roll over its edge. In fact, if we assign a coefficient of restitution of $\epsilon=0.87$ to the cube equation in (21), we find that the impulse per travelled distance closely matches the most stable disc performances as shown by the blue dashed line. We can therefore claim that the cube would have to conserve $87 \%$ of its velocity at every collision in order to keep up with the off-centred disc performance. In a next step we will examine if this observation can be replicated in a real-life experiment.

\section{Roвот DESIGN}

We designed and built a robot named Robbit specifically to operate close to the theoretical ideal. There are three main design challenges, namely the impulsive actuation, the reduction of moment of inertia of the main structure, and the extension of the planar model assumption in the real, three dimensional world. Parameter values are in accordance with table I for inertial and geometrical parameters.

Our main actuation on the robot is a 100W Maxon ${ }^{\circledR}$ EC 60 flat motor, torque controlled with a Maxon ${ }^{\circledR}$ EPOS2 controller which is not on the robot but placed away from it and connected with wires. The braking mechanism is operated with a standard Parallax ${ }^{\circledR}$ servo motor. The design of the impulsive

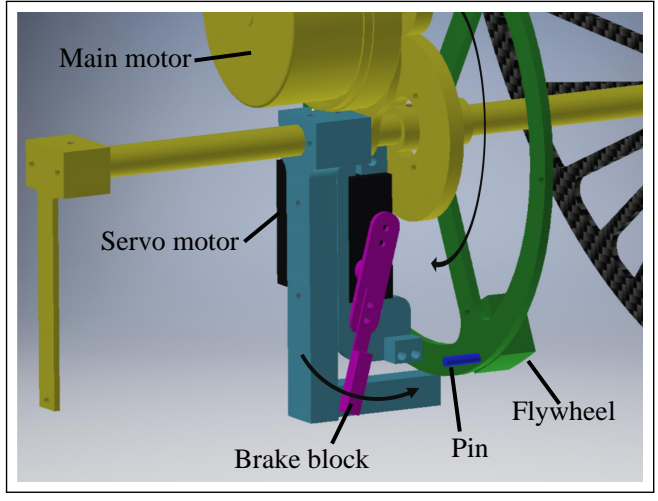

Fig. 8: Mechanism of the brake for impulse transmission. The rotation of the flywheel is stopped by an aluminium brake block which is moved by the servo motor to close the connection between flywheel pins and the main robot structure.

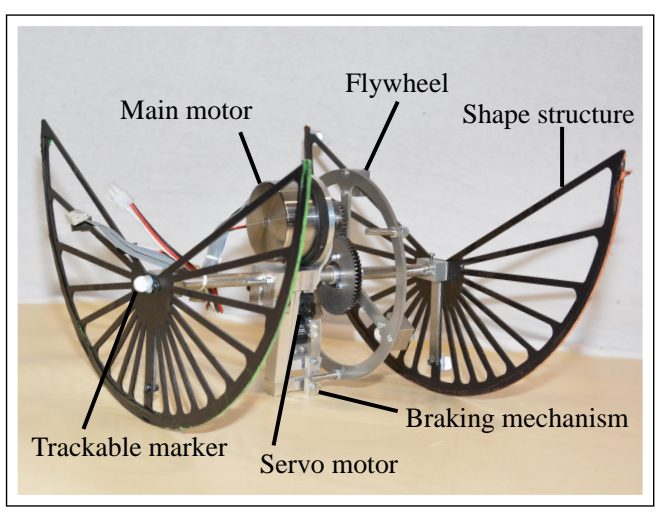

Fig. 9: The off-centred disc robot Robbit.

angular actuation uses a similar mechanism as presented in [10]. As shown in Figure 8, our main actuator is fixed on the main body of the robot and is transmitting its torque with a gear reduction of 2.4 to the flywheel which is rotating around the main steel shaft of the robot. The rotation velocity of the main motor relative to the robot is registered by the motor controller with hall-sensors and a rotary encoder. Once the desired rotation speed of the flywheel is reached, the braking mechanism is activated. Three steel pins firmly attached to the flywheel are passing through a gap in the main robot structure each once per revolution. A small aluminium plate is attached to the servo motor, which can be moved across the gap upon servo motor activation. This blocks the pins from moving through the gap and causes the angular momentum to be exchanged between the flywheel and the gap structure. Another aluminium structure then transfers the impulse to the main steel shaft, inducing a rotation of the whole robot.

For reduction of the moment of inertia, we chose a carbon fibre sheet of $2 \mathrm{~mm}$ thickness with a spoked wheel-like structure. To mimic the planar dynamics in the physical model, we designed the robot symmetrically in its transversal dimension, connecting the two carbon fibre semi-discs with an aluminium rod of $200 \mathrm{~mm}$ length. 


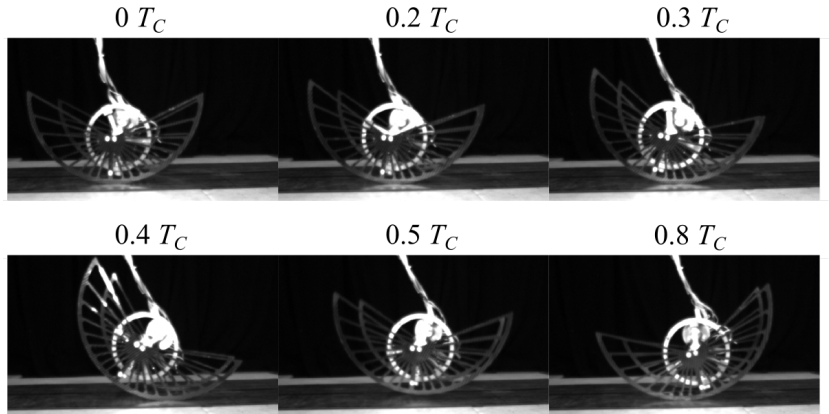

Fig. 10: Series of pictures of Robbit over one gait cycle $T_{C}$ from slow motion recordings $(300 \mathrm{~Hz})$.

\section{EXPERIMENTAL RESULTS}

To test Robbit, we prepared a locomotion track with an OptiTrack ${ }^{\circledR}$ motion capturing system which recorded four trackable markers rigidly fixed on the robot with a framerate of $250 \mathrm{~Hz}$. The system is controlled centrally with a desktop PC running Matlab ${ }^{\circledR}$ from which the torque targets are sent to the motor controller, encoder signals are being evaluated for a braking decision, and the braking signal is being sent via a COM-port to an Arduino ${ }^{\circledR}$ micro-controller which operates the servo motor. The robot starts at rest and a constant torque is applied until the defined impulse-limit is reached, which caused the braking mechanism to be activated. The motor torque is then set to zero for $100 \mathrm{~ms}$ during flight phase, before it is again set to the nominal torque. We ran the experiment for $6 \mathrm{~s}$ and recorded the trajectory of the trackable markers.

Figure 10 illustrates the robot's motion with a series of pictures taken over one period of the locomotion gait. The period time $T_{C}$ is around $0.3 \mathrm{~s}$. During $0.4 T_{C}$ of the cycle, the main motor is accelerating the flywheel, which causes the main structure to roll over its carbon fibre feet. Once the predefined impulse limit is reached, the servo motor engages the brake, which induces a momentum transfer between the flywheel and the main robot body. The picture at $0.5 T_{C}$ shows the robot in mid-flight phase, where the main motor torque is set to zero and the brake is disengaged by the servo motor. The cycle then ends with the beginning of the next stance phase. As can be seen from the series of pictures, this cycle results in a net forward motion of Robbit. See also the supplementary material for a slow motion video of the robot motion.

The result of the centre of mass trajectories for the generalised coordinates $\boldsymbol{q}$ are shown in Figure 11, excluding the transient phase. The control corresponds to a torque of $0.16 \mathrm{Nm}$ and an impulse limit that is reached at a relative angular velocity of the flywheel and main structure of $58 \frac{\mathrm{rad}}{\mathrm{s}}$. The figure shows the centre of mass trajectories for seven consecutive hops as a function of the cycle progress in percent. We compare the experimental result to the prediction of the simulation framework described in section III-C. We see that the simulation predicts the magnitude of displacement over the full gait cycle, as well as the observed double hump in the vertical position coordinate. The second hump is due to the backward rotation after touchdown, lifting the centre of
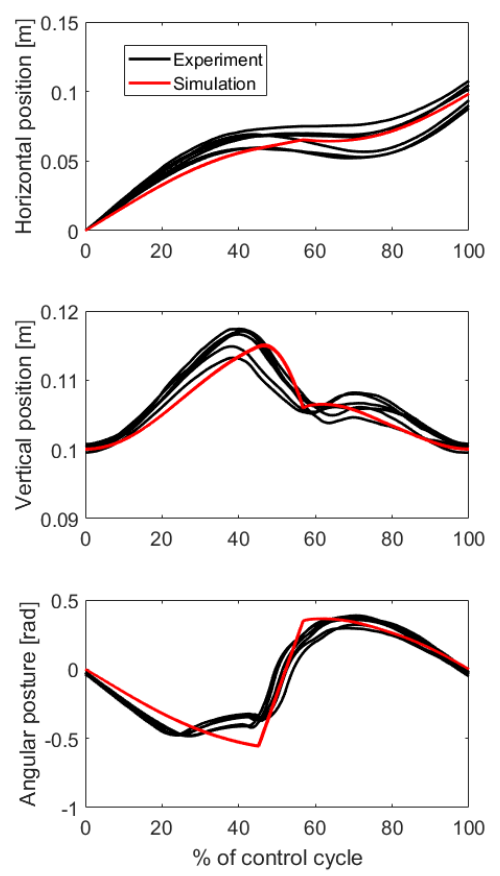

Fig. 11: Centre of mass trajectories of generalised coordinates $\boldsymbol{q}=[x, y, \phi]$ of experiment and simulation excluding the transient phase. The horizontal axis captures one full control cycle. The black lines correspond to seven consecutive hops in experiment, and the red line shows the simulation result with the same actuation parameters as in the experiment for ten consecutive hops ( $T=0.16 \mathrm{Nm}$ and $\left.d \zeta_{\text {lim }}=0.059 \mathrm{Nms}\right)$.

mass first before the rolling direction changes, which can be observed in both simulation and real-world experiment.

We controlled the robot with a range of control parameters, as shown in Table I under Experiment control. The velocities of these experiments as a function of the impulse per travelled distance (derived from impulse limit and the measured hopping frequency) are shown in Figure 12, together with a fit to the most impulse effective stable solutions found in simulation and the theoretical cube performance. The results show, in accordance with the theoretical predictions, that the impulse per distance is roughly ten times lower than the cube's theoretical prediction. Almost all of the experimentally recorded trajectories correspond to trajectory type 4 in Figure 5b, which are stable, efficient, and characterised by a long stance phase followed by a rather short flight phase. The experiments further show that any configuration of control parameters $\beta$ leads to bounded and almost periodic locomotion after a few transient hops. The system therefore not only outperforms a cube in its economical use of impulses, but also converges to periodic and continuous locomotion without precisely defined control parameters. The system finds its fixed-point autonomously for the given control, a behaviour which we ascribe to the selfstabilising properties of the strictly convex shape.

\section{DISCUSSION}

The results showed, based on the cases of a disc with offcentred mass and a cube, that strictly convex shapes outper- 


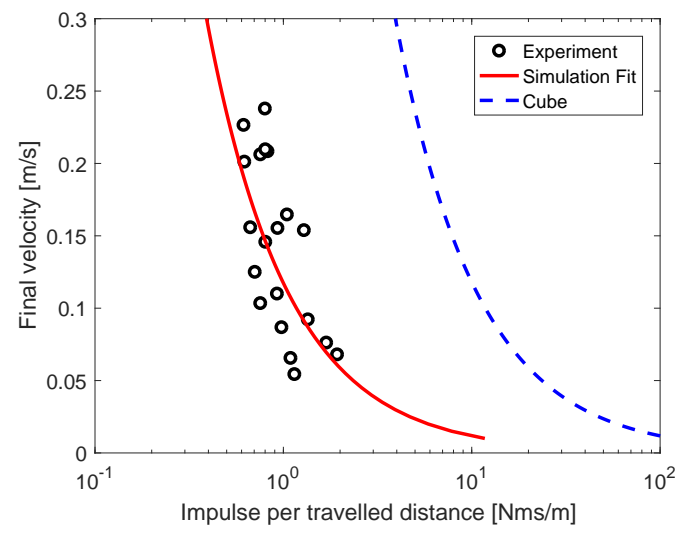

Fig. 12: Experimental converged hopping speeds as a function of impulse per travelled distance. Each circle indicates a robot experiment with fixed actuation parameters. We set the parameter range for the series of experiments for torque between 0.1 and $0.3 \mathrm{Nm}$ and impulse limit between 0.04 and $0.07 \mathrm{Nms}$. Experiment duration was 6 seconds and the data plotted corresponds to the average speed as a function of the average impulses over the travelled distance. The red solid line corresponds to a fit of the most impulse effective stable solutions obtained from simulation, and the dashed blue line corresponds to the theoretical speed of an impulsively actuated cube with face diagonal $l=2(R-a)$ and coefficient of restitution $\epsilon=0$.

form theoretical performance of non-strictly convex shapes in terms of their locomotion speed as a function of impulse per travelled distance. By comparing systems in terms of their impulse per travelled distance at constant speeds, we essentially compare their energetic costs of transport. The dimensionless cost of transport as defined by [20] for locomotion in animals, is the ratio of metabolic rate to the product of speed and body weight. This definition has been adopted by the robotics community, such as in [21], by replacing metabolic cost with energy used. Animals reduce their energy use with mechanisms to efficiently redirect their centre of mass velocity [22]. Among others, two important mechanisms have been thoroughly studied in biomechanics and robotics, namely spring-mass models [23] and inverted pendulum models [24]. Both mechanisms conserve a fraction of the kinetic energy from the previous step to the next. In the case of strictly convex shapes, energy is conserved by smoothly redirecting the centre of mass through a rolling motion. This idea has been considered as a limit case for legged locomotion with infinitely many legs [25]. Loss-less redirection of centre of mass is necessary to minimise the cost of transport, but it is not sufficient. A significant source of energy loss in terrestrial locomotion occurs due to interactions with the ground. As we showed in Figure 7, a cube would have to restore $87 \%$ of its velocity at touchdown to perform with the same cost of transport as the off-centred disc for a given locomotion speed, which indicates a low collisional cost at touchdown in the strictly convex shape case. This observation agrees well with studies of bipedal walking with curved feet, as the authors describe a reduction in energy loss in the step-to-step transition compared to point feet [26], [27], [28].

In this work, we have also demonstrated how strictly convex shapes can lead to stable hopping locomotion without any state feedback except the flywheel speed. The self-stabilising properties of curved feet have been studied for locomotion in monopods, bipeds, and quadrupeds [14]. Many other examples of self-stabilising locomotion exist [15], [6], but the applications move beyond locomotion; related work in the stabilisation of juggling through a convex shape [29] showed how apex and paddle position can be self-stabilised, meaning no sensory feedback is required to juggle a ball with a paddle. This relates to our hopping system given a fixedpoint actuation $\beta^{*}$, as no feedback from the environment is necessary to stabilise the motion, not even to transition from resting position to the final locomotion speed. An extension of this work could include temporal change of shape to further improve the cost of transport or locomotion speed. The potential of active external shape is abundant in robotics. There are examples of robots that use their strictly convex shape to actively propel themselves, as shown in the modular loop robot presented in [30], or in the spherical robot with inflatable pouches that induce a rolling motion, as studied in [31]. We have recently shown that a shape change during locomotion which does no positive work on the system can indeed increase locomotion speed [32]. For future investigations of shape and its effect on locomotion performance, we are planning to use and study the defined contact function as presented in this work. The contact function defines the constraint forces and is therefore determining the passive dynamic behaviour which may help understand self-stability in this system.

Our work showed how locomotion with inelastic impacts can be considerably improved in terms of energy used for locomotion. Further improvement can be expected when elastic restitution is used to recover more kinetic energy at each collisional event. This could be achieved by adapting the structure and material of the disc and we will study this in future investigations. Improved energy usage is especially useful in exploratory ventures where energy is scarce, as is the case in space robotics, such as in [19]. Furthermore, our locomotion method can provide a new mode of locomotion for the vast number of spherical robots [33] which have been built and studied in recent years. For instance, a spherical robot with internal momentum-stored impulse actuation and off-centred mass can generate the described hopping motion, which would enhance its locomotion capabilities. We are currently investigating the role of moment of inertia and eccentricity on the energy effectiveness to further improve the locomotion capabilities of such systems.

Our findings, shown in Figure 7, indicate that stability and efficiency are coupled such that the fixed-points associated with the smallest maximal eigenvalue of their return map are also the ones which demand the smallest impulse per travelled distance. We have presented similar findings of the coupling between stability and efficiency in [6], and also for the case of a hopping robot with a strictly convex foot shape. It is a striking feature of these locomotive systems that they are both efficient and robust. 


\section{CONCLUSION}

In this paper, we studied the locomotion of strictly convex shapes actuated by momentum-stored impulses. We proved for the simple case of a disc with an off-centred mass, that fixedpoints to this locomotion methods exist, and that a large number of them are stable. A simulation of the system from resting position reveals the superiority in terms of required impulse per travelled distance, as compared to the case of an impulseactuated cube. We validated our findings in a real world platform with the robot Robbit, showing that ten times lower impulses are required to travel a certain distance compared to the theoretical case of a cube. The results demonstrate the significance of shape in the dynamical behaviour of hopping, and how it can be used to improve stability and efficiency of locomotion in robots.

\section{APPENDIX}

The four derivatives in (18) have to be computed with care, as some of the occurring partial derivatives do not address quantities that are given in an analytic explicit expression, but rather by a differential equation or an implicit equation. To dissect the expressions we can rewrite (18) to

$$
\mathbf{A}=\left.\left[\begin{array}{cc}
\frac{d \Gamma_{1}}{d \phi_{T D}} & \frac{d \Gamma_{1}}{d \dot{\phi}_{T}^{+} D} \\
\frac{d \Gamma_{2}}{d \phi_{T D}} & \frac{d \Gamma_{2}}{d \dot{\phi}_{T D}^{+}}
\end{array}\right]\left[\begin{array}{ll}
K_{1 \phi} & K_{2 \dot{\phi}} \\
K_{1 \phi} & K_{2 \dot{\phi}}
\end{array}\right]\right|_{\phi_{0}, \dot{\phi}_{0}} .
$$

with the suffix $T D$ indicating the touchdown state and with

$$
\begin{aligned}
K_{1 \phi}:= & {\left.\left[\frac{\partial \phi_{T D}}{\partial \phi}+\frac{d \phi_{T D}}{d \dot{\phi}_{T O}^{+}}\left(\frac{\partial \dot{\phi}_{T O}^{+}}{\partial \phi}+\frac{d \dot{\phi}_{T O}^{+}}{d \dot{\phi}_{T O}^{-}} \frac{d \dot{\phi}_{T O}^{-}}{d \phi}\right)\right]\right|_{\phi_{0}, \dot{\phi}_{0}}, } \\
K_{1 \dot{\phi}}:= & {\left[\frac{\partial \dot{\phi}_{T D}^{+}}{\partial \phi}+\frac{d \dot{\phi}_{T D}^{+}}{d \dot{\phi}_{T D}^{-}}\left(\frac{\partial \dot{\phi}_{T D}^{-}}{\partial \phi}+\right.\right.} \\
& \left.\left.\frac{d \dot{\phi}_{T D}^{-}}{d \dot{\phi}_{T O}^{+}}\left(\frac{\partial \dot{\phi}_{T O}^{+}}{\partial \phi}+\frac{d \dot{\phi}_{T O}^{+}}{d \dot{\phi}_{T O}^{-}} \frac{d \dot{\phi}_{T O}^{-}}{d \phi}\right)\right)\right]\left.\right|_{\phi_{0}, \dot{\phi}_{0}}, \\
K_{2 \phi}:= & {\left.\left[\frac{\partial \phi_{T D}}{\partial \dot{\phi}}+\frac{d \phi_{T D}}{d \dot{\phi}_{T O}^{+}}\left(\frac{\partial \dot{\phi}_{T O}^{+}}{\partial \dot{\phi}}+\frac{d \dot{\phi}_{T O}^{+}}{d \dot{\phi}_{T O}^{-}} \frac{d \dot{\phi}_{T O}^{-}}{d \dot{\phi}}\right)\right]\right|_{\phi_{0}, \dot{\phi}_{0}}, }
\end{aligned}
$$

and

$$
\begin{aligned}
K_{2 \dot{\phi}}:= & {\left[\frac{\partial \dot{\phi}_{T D}^{+}}{\partial \dot{\phi}}+\frac{d \dot{\phi}_{T D}^{+}}{d \dot{\phi}_{T D}^{-}}\left(\frac{\partial \dot{\phi}_{T D}^{-}}{\partial \dot{\phi}}+\right.\right.} \\
& \left.\left.\frac{d \dot{\phi}_{T D}^{-}}{d \dot{\phi}_{T O}^{+}}\left(\frac{\partial \dot{\phi}_{T O}^{+}}{\partial \dot{\phi}}+\frac{d \dot{\phi}_{T O}^{+}}{d \dot{\phi}_{T O}^{-}} \frac{d \dot{\phi}_{T O}^{-}}{d \dot{\phi}}\right)\right)\right]\left.\right|_{\phi_{0}, \dot{\phi}_{0}} .
\end{aligned}
$$

Computation of most of the terms in (22) are straight forward to calculate through (11) to (15). Special attention however is required for the left-hand side matrix in (22), as these derivatives are effectively influencing the result of an integration rather than a closed form expression. To calculate the derivatives, we define the sensitivity with respect to our parameters of interest $p_{1}=\phi_{T D}$ and $p_{2}=\dot{\phi}_{T D}$ as $\boldsymbol{r}_{i}=\frac{d \boldsymbol{z}}{d p_{i}}$. Through differentiation of (15) by $p_{i}$, we obtain

$$
\dot{\boldsymbol{r}}_{i}=\frac{d \boldsymbol{f}}{d \boldsymbol{z}} \boldsymbol{r}_{i}+\frac{d \boldsymbol{f}}{d p_{i}}, \quad i \in\{1,2\},
$$

where $f$ is the right-hand side of (15) and the last term drops out since $f$ does not depend on the post-touchdown states. Solving this ODE with initial conditions $\boldsymbol{r}_{1}\left(\boldsymbol{z}_{0}\right)=(1,0)^{T}$ and $\boldsymbol{r}_{2}\left(\boldsymbol{z}_{0}\right)=(0,1)^{T}$ until $z_{1}=\phi_{0}$, will provide all entries in the left-hand side matrix in (22) for the case of an open-loop controlled system. However, our controller is stopping not as a function of time, but as a function of the impulse $d \zeta$. In other words, in the shown sensitivity analysis, we did not account for the effect that a disturbance has on the stance phase time $t_{s}$. To include this, we first express (15) as a function of $d \zeta$ rather than $t$. This requires a change of variable with

$$
\frac{d \boldsymbol{z}}{d t}=\frac{d \boldsymbol{z}}{d(d \zeta)} \frac{d(d \zeta)}{d t}
$$

and therefore get

$$
\frac{d \boldsymbol{z}}{d(d \zeta)}=\frac{\dot{\boldsymbol{z}}}{(\dot{d} \zeta)}=\frac{\boldsymbol{f}}{(\dot{d} \zeta)}
$$

The derivative of the impulse can be derived from (8) to (9)

$$
(\dot{d} \zeta)=\frac{I_{S} I_{R}}{I_{S}+I_{R}}\left(\frac{T}{t_{s}}-\dot{z}_{2}\right) .
$$

It remains to change the coordinates in (27) in terms of impulse instead of time

$$
\frac{d \boldsymbol{r}_{i}}{d(d \zeta)}=\left(\frac{1}{(\dot{d} \zeta)} \frac{d \boldsymbol{f}}{d \boldsymbol{z}}-\frac{\boldsymbol{f}}{(\dot{d} \zeta)^{2}} \frac{(\dot{d} \zeta)}{d \boldsymbol{z}}\right) \boldsymbol{r}_{i} .
$$

The solution of equation (31) now truly leads to the entries of the left-hand side of (22) for our control method, as we can simply integrate to the impulse limit $d \zeta_{\text {lim }}$. Note that in (31) we assume knowledge of $z(d \zeta)$. If this is not given, the system has to be augmented to a system of four rather than two ODEs, where the additional ODEs are given by (29).

The derivatives which derive post-flight phase states require attention, as the equations of the flight time are only given implicitly. More specifically the terms $\frac{\partial \phi_{T D}}{\partial \phi_{0}}$ and $\frac{\partial \phi_{T D}}{\partial \dot{\phi}_{T O}^{+}}$are

$$
\begin{aligned}
\frac{\partial \phi_{T D}}{\partial \phi_{0}} & =\frac{\partial}{\partial \phi_{0}}\left(\phi_{0}+\dot{\phi}_{T O}^{+} t_{F}\left(\phi_{0}, \dot{\phi}_{T O}^{+}\right)\right) \\
& =1+\dot{\phi}_{T O}^{+} \frac{d t_{F}}{d \phi_{0}}
\end{aligned}
$$

and accordingly

$$
\frac{\partial \phi_{T D}}{\partial \dot{\phi}_{T O}^{+}}=t_{F}+\dot{\phi}_{T O}^{+} \frac{d t_{F}}{d \dot{\phi}_{T O}^{+}} .
$$

To find the derivatives of the flight time $t_{F}$, we simply take the derivative of the implicit equation (12) and solve for $\frac{d t_{F}}{d \phi_{0}}$ and $\frac{d t_{F}}{d \dot{\phi}_{T O}^{+}}$, which yields

$$
\frac{d t_{F}}{d \phi_{0}}=\frac{-a \sin \phi_{0}-\dot{\phi}_{T O}^{+} a t_{F} \cos \phi_{0}+a \sin \left(\phi_{0}+\dot{\phi}_{T O}^{+} t_{F}\right)}{\dot{\phi}_{T O}^{+} a \sin \phi_{0}-g t_{F}-a \dot{\phi}_{T O}^{+} \sin \left(\phi_{0}+\dot{\phi}_{T O}^{+} t_{F}\right)},
$$

and

$$
\frac{d t_{F}}{d \dot{\phi}_{T O}^{+}}=\frac{-a t_{F} \sin \phi_{0}+a t_{F} \sin \left(\phi_{0}+\dot{\phi}_{T O}^{+} t_{F}\right)}{\dot{\phi}_{T O}^{+} a \sin \phi_{0}-g t_{F}-a \dot{\phi}_{T O}^{+} \sin \left(\phi_{0}+\dot{\phi}_{T O}^{+} t_{F}\right)} .
$$

We used the relation $y=R-a \cos \phi_{0}$ and $\dot{y}=\dot{\phi}_{T O}^{+} a \cos \phi_{0}$ to replace the vertical position and velocity components in (12). The remaining terms of (22) are easily calculated. 


\section{ACKNOWLEDGMENT}

The authors would like to thank Alistair Ross and Adam Boyce for his support in building Robbit, and Trisha Banerjee for editing the manuscript. This project was supported by the UK's Engineering and Physical Sciences Research Council (EPSRC).

\section{REFERENCES}

[1] M. H. Raibert, "Legged robots that balance", MIT press, 1989.

[2] M. Raibert, K. Blankespoor, G. Nelson, and R. Playter, "Bigdog the rough-terrain quadruped robot," in Proc. Int. Fed. Autom. Contr., 2008, pp. $10822-10825$.

[3] R. M. Alexander, "Energy-saving mechanisms in walking and running," J. Exp. Biol., vol. 160, no. 1, pp. 55-69, 1991.

[4] J. M. Winters, "Hill-based muscle models: a systems engineering perspective," Multiple muscle systems, Springer New York, pp. 69-93, 1990.

[5] G.A. Pratt, and M. M. Williamson, "Series elastic actuators," in Proc. IEEE Int. Conf. Intel. Robot. Sys., 1995, pp. 399-406.

[6] F. Gunther, F. Giardina, and F. Iida "Self-stable one-legged hopping using a curved foot," in Proc. IEEE Int. Conf. Robot. Autom., 2014, pp. 5133-5138.

[7] M. Kovac, M. Fuchs, A. Guignard, J. C. Zufferey, and D. Floreano, "A miniature $7 \mathrm{~g}$ jumping robot," in Proc. IEEE Int. Conf. Robot. Autom., 2008, pp.373-378.

[8] D. W. Haldane, M. M. Plecnik, J. K. Yim, and R. S. Fearing, "Robotic vertical jumping agility via series-elastic power modulation," Science Robotics, vol. 1, no. 1, 2016.

[9] J. Burdick, and P. Fiorini, "Minimalist jumping robots for celestial exploration," Int. J. Robot. Res., vol. 22, no. 7-8, pp. 653-674, 2003.

[10] M. Gajamohan, M. Merz, I. Thommen, and R. D'Andrea, 'The cubli: A cube that can jump up and balance," in Proc. IEEE Int. Conf. Intel. Robot. Sys., 2012, pp.3722-3727.

[11] B. J. Hockman, A. Frick, R. G. Reid, I. A. Nesnas, and M. Pavone, "Design, Control, and Experimentation of Internally Actuated Rovers for the Exploration of Low gravity Planetary Bodies," J. Field Robot., vol. 34, no. 1, pp. 5-24, 2017.

[12] J. W. Romanishin, K. Gilpin, S. Claici, and D. Rus, "3D M-Blocks: Self-reconfiguring robots capable of locomotion via pivoting in three dimensions," in Proc. IEEE Int. Conf. Robot. Autom., 2015, pp.19251932.

[13] Boston Dynamics, SandFlea Jumping Robot, retrieved at https://www.bostondynamics.com/sandflea.

[14] R. Ringrose, "Self-stabilizing running," in Proc. IEEE Int. Conf. Robot. Autom. 1997, pp. 487-493.

[15] F. Guenther, and F. Iida, "Energy-Efficient Monopod Running With a Large Payload Based on Open-Loop Parallel Elastic Actuation,” IEEE Trans. Robot., vol. 33, no. 1, pp. 102-113, 2017.

[16] W. J. Stronge, "Rigid body collisions with friction," In P. R. Soc. A, vol. 431, no. 1881, pp. 169-181, 1990.

[17] C. Glocker, and C. Studer, "Formulation and preparation for numerical evaluation of linear complementarity systems in dynamics," Multibod. Syst. Dyn., vol. 13, no. 4, pp. 447-463, 2005.

[18] R. I. Leine, D. H. Van Campen, and C. Glocker. "Nonlinear dynamics and modeling of various wooden toys with impact and friction." Modal Analysis, vol. 9, no. 1-2, pp 25-78, 2003.

[19] R. Allen, M. Pavone, C. McQuin, I. A. Nesnas, J. C. Castillo-Rogez, T. N. Nguyen, and J. A. Hoffman, "Internally-actuated rovers for all-access surface mobility: Theory and experimentation," in Proc. IEEE Int. Conf. Robot. Autom., 2013, pp. 5481-5488.

[20] V.A. Tucker, "Energetic cost of locomotion in animals," Comp. Biochem. Physiol., vol. 34, no. 4, pp. 841-846, 1970.

[21] S. Collins, A. Ruina, R. Tedrake, and M. Wisse, "Efficient bipedal robots based on passive-dynamic walkers," Science, vol. 307, no. 5712, pp. $1082-1085,2005$

[22] G.A. Cavagna, N.C. Heglund and C.R. Taylor, "Mechanical work in terrestrial locomotion: two basic mechanisms for minimizing energy expenditure," Am. J. Physiol. Regul. Integr. Comp. Physiol., vol. 233, no. 5 , pp. $243-261,1977$

[23] R. Blickhan R, "The spring-mass model for running and hopping," $J$. Biomech., vol. 22, no. 11-12, pp. 1217-1227, 1989.
[24] S. Kajita, F. Kanehiro, K. Kaneko, K. Yokoi, and H. Hirukawa, "The 3D Linear Inverted Pendulum Mode: A simple modeling for a biped walking pattern generation," in Proc. IEEE Int. Conf. Intel. Robot. Sys., 2001, pp. 239-246.

[25] A. Ruina, J. E. Bertram, and M. Srinivasan, "A collisional model of the energetic cost of support work qualitatively explains leg sequencing in walking and galloping, pseudo-elastic leg behavior in running and the walk-to-run transition," J. Theor. Biol., vol. 237, no.2, pp. 170-192, 2005.

[26] P. G. Adamczyk, and A. D. Kuo, "Mechanical and energetic consequences of rolling foot shape in human walking," J. Exp. Biol., vol. 216, no. 14, pp. 2722-2731, 2013.

[27] F. Asano, Z.W. Luo, "The effect of semicircular feet on energy dissipation by heel-strike in dynamic biped locomotion," in Proc. IEEE Int. Conf. Robot. Autom., 2007, pp.3976-3981.

[28] A. E. Martin, D. C. Post, J. P. Schmiedeler, ”Design and experimental implementation of a hybrid zero dynamics-based controller for planar bipeds with curved feet," Int. J. Robot. Res., vol. 33, no. 7, pp. 9881005 , 2014.

[29] P. Reist, and R. D'Andrea, "Design and analysis of a blind juggling robot," IEEE Trans. Robot., vol. 28, no. 6, pp. 1228-1243, 2012.

[30] J. Sastra, S. Chitta, and M. Yim, "Dynamic rolling for a modular loop robot," Int. J. Robot. Res., vol. 28, no. 6, pp. 758-773, 2009.

[31] K. W. Wait, P. J. Jackson, and L. S. Smoot, "Self locomotion of a spherical rolling robot using a novel deformable pneumatic method," in Proc. IEEE Int. Conf. Robot. Autom., 2010, pp. 3757-3762.

[32] F. Giardina, and F. Iida, "Discrete Foot Shape Changes Improve Dynamics of a Hopping Robot," in Proc. Int. Symp. Exp. Robot., 2016, pp. 113-122.

[33] R. Chase, and A. Pandya "A review of active mechanical driving principles of spherical robots," Robotics vol. 1 no. 1, pp. 3-23, 2012.

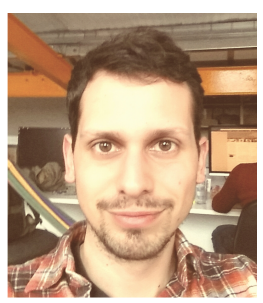

Fabio Giardina received the B.Sc. and M.Sc. degrees in mechanical engineering from the Swiss Federal Institute of Technology Zurich, Zurich, Switzerland, in 2010, and 2012, respectively. He is currently working toward the $\mathrm{PhD}$ degree in the Machine Intelligence group in the Biologically Inspired Robotics Laboratory, University of Cambridge, Cambridge, U.K. His research interests include discrete control and the effects of morphology on dynamics of robotic locomotion systems.

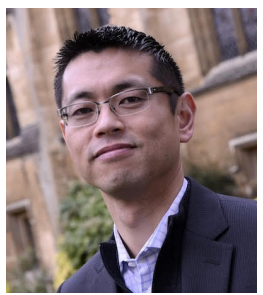

Fumiya Iida (SM'17) is a university lecturer at Department of Engineering, University of Cambridge. $\mathrm{He}$ is also the director of Biologically Inspired Robotics Laboratory and a fellow of Corpus Christi College. He received his bachelor and master degrees in mechanical engineering at Tokyo University of Science in Japan, and Dr. sc. nat. in Informatics at University of Zurich in Switzerland. During his $\mathrm{PhD}$ project, he was also engaged in biomechanics research of human locomotion at Locomotion Laboratory, University of Jena in Germany. While he worked as a postdoctoral associate at the Computer Science and Artificial Intelligence Laboratory, Massachusetts Institute of Technology in USA, he was awarded the Fellowship for Prospective Researchers from the Swiss National Science Foundation, and then, the Swiss National Science Foundation Professorship hosted by ETH Zurich. In 2014 he moved to the University of Cambridge as the director of Bio-Inspired Robotics Laboratory. His research interest includes biologically inspired robotics, embodied artificial intelligence, and soft robotics, where he was involved in a number of research projects related to robot locomotion, manipulation, and human-robot interactions leading to some start-up companies. He was a recipient of the IROS2016 Fukuda Young Professional Award, and Royal Society Translation Award in 2017 